SCIENTIFIC LETTER

\title{
Transoesophageal ultrasonography: a new approach to imaging the thoracic aorta
}

\author{
J B Chambers, P R Taylor, J F Reidy, C Woods, S J Carter, T S Padayachee
}

Heart 2005;91:245-246. doi: 10.1136/hrt.2004.036590

$\mathrm{T}$ ransoesophageal echocardiography (TOE) is often the initial investigation of choice for imaging acute and chronic aortic disease including dissection and ectasia. It is also used in the placement of endovascular stent grafts, to monitor deployment of the stent, and to assess results. ${ }^{1}$ It is ideally suited because of the close proximity of the oesophageal imaging window to the arch and descending thoracic aorta. However, endovascular stent grafting is increasingly being performed under regional and local anaesthesia and it is impossible to tolerate a TOE probe for the length of the procedure. We therefore investigated the feasibility of imaging the thoracic aorta using a device originally developed for intracardiac echocardiography.

\section{PATIENTS AND METHODS}

The ultrasound-tipped catheter (AcuNav, Siemens, Mountain View California) is $90 \mathrm{~mm}$ long and $3.2 \mathrm{~mm}(10 \mathrm{~F})$ in diameter compared with $9 \mathrm{~mm}(27 \mathrm{~F})$ for a standard TOE probe. The imaging range is from $5.5-10 \mathrm{MHz}$ and it has colour (5-7 MHz) and pulsed Doppler (4-5 MHz) capabilities. There is only one imaging plane, but transverse and intermediate views are possible because the tip of the catheter has four-direction articulation enabling both anteroposterior and left-right views. We obtained approval for using the AcuNav transoesophageally from the local hospital ethics committee and each patient gave written consent. We studied eight patients; five male and three female, aged $42-83$ years. One was studied preoperatively, five during surgery, and four at a mean of four months after surgery. Stenting was performed for aortic dissection $(\mathrm{n}=3)$ and thoracic aneurysms $(\mathrm{n}=5)$.

The patient was asked to sniff and the least congested nostril chosen. Cocaine paste (25\%) was applied to the nasal passage using a cotton bud and xylocaine $(4 \%)$ was sprayed to the back of the nose and over the oropharynx. Tape was placed $50 \mathrm{~cm}$ from the probe tip to act as a marker. The probe was introduced through the nose with mild fixed flexion and then the lock taken off. The patient was then asked to swallow, sometimes aided by sips of water, and the probe was advanced gently up to the $50 \mathrm{~cm}$ marker. The probe remained in position for approximately 20 minutes (range 15 minutes to three hours).

\section{RESULTS}

The probe was well tolerated by all patients and there were no complications. The descending aorta and the underlying
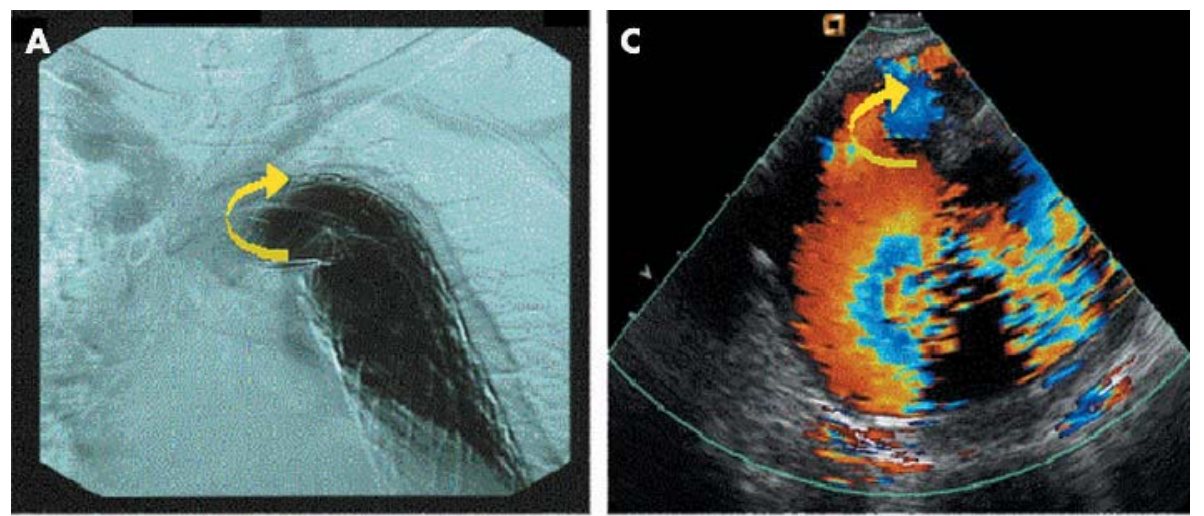

Figure 1 Imaging of the thoracic aorta three months atter endoluminal stenting for type B dissection. Angiograms demonstrating the proximal (A) and distal (B) ends of the stent graft with extra-luminal contrast of uncertain origin and direction. Transoesophageal ultrasonography using colour flow mapping clearly identified both a proximal (C) and distal (D) endoleak.
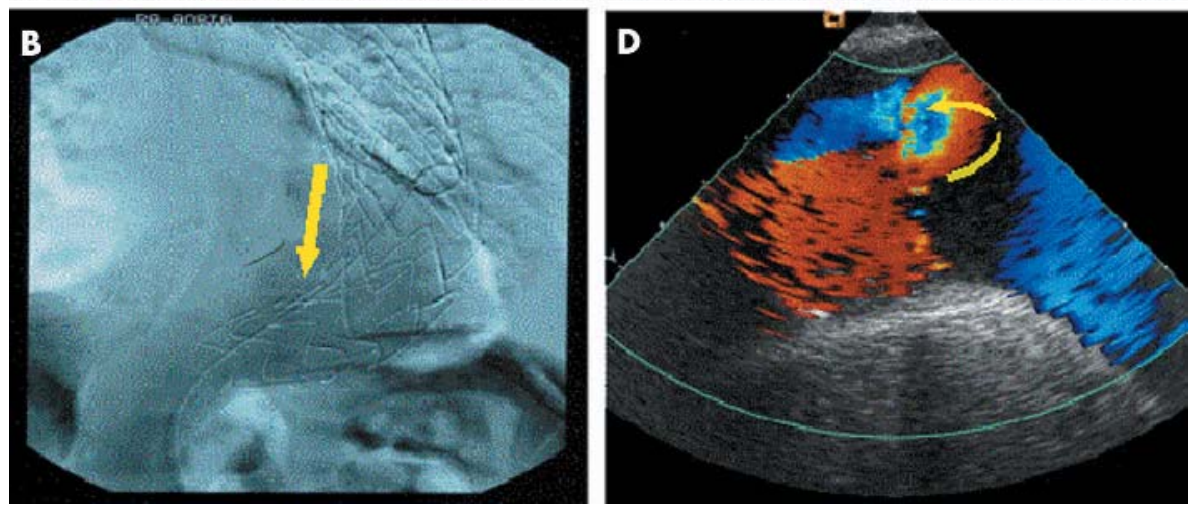
pathology was confirmed in all patients. The aortic arch was obscured in most instances, although the left subclavian origin was demonstrated. Before stenting the anatomy of the normal aorta, where the ends of the stent graft were to be fixed, was demonstrated in four of the five pre-stent scans. During the endovascular procedure, the ultrasound image was lost immediately after deployment presumably as a result of air within the structure of the graft material (polytetrofluroethylene). However, the stent graft could be imaged in all patients studied late after deployment. We were able to confirm the position of the stent, thrombosis of the aneurysm, or false lumen and incomplete stent wall apposition. In a patient with extra-luminal contrast on computerised tomography and angiography (fig 1A, B), we were able to show endoleaks from both the proximal and distal ends of the stent (fig IC, D).

\section{DISCUSSION}

The AcuNav has been used for TOE imaging in animals, ${ }^{23}$ infants, ${ }^{4}$ and in one preliminary report in adults. ${ }^{5}$ This is the first report of its use for imaging endovascular treatment for thoracic aortic disease in humans. The main advantage over conventional TOE is its tolerability, making it suitable for monitoring for relatively long periods. It also has superior resolution to conventional TOE as a result of the higher carrier frequencies. For all ultrasound techniques, there is a trade-off between resolution and penetration, but with this technique we have now shown good views of the descending thoracic aorta. Its ability to scan in multiple planes is limited by the possession of only one imaging plane although the manoeuvrability of the tip to some degree compensates for this deficiency. Heat generation at the tip is a theoretical problem; although limited exposures have been shown to induce no more heating than conventional $\mathrm{TOE}^{2}$ it is probably wise to limit the use of colour Doppler, which generates more heat than other modalities, and also to stop imaging when not essential. The cost is currently high since each probe costs $£ 1400$ and is only licensed for single use intravascularly. However, such probes have been sterilised and re-used ${ }^{5}$ although it is not known how often this can be done.

In conclusion, this is a promising technique which requires formal prospective validation against other techniques, notably TOE, computerised tomography, magnetic resonance, and contrast angiography. The question of re-use also needs discussion to limit its considerable cost. Preliminary results suggest that it will complement the use of cross sectional imaging by showing flow information in real time, while three dimensional imaging may be feasible in the future and will lend further weight to the value of this technique.

\section{Authors' affiliations}

J B Chambers, Department of Adult Echocardiography, Guy's \& St Thomas' Hospitals, London, UK

P R Taylor, Department of Surgery, Guy's \& St Thomas' Hospitals J F Reidy, Department of Radiology, Guy's \& St Thomas' Hospitals C Woods, Department of Anaesthetics, Guy's \& St Thomas' Hospitals S J Carter, T S Padayachee, Department of Ultrasonic Angiology, Guy's \& St Thomas' Hospitals

Correspondence to: Dr T S Padayachee, Department of Ultrasonic Angiology, Guy's Hospital, St Thomas Street, London SE91 9RT, UK; soundrie.padayachee@kcl.ac.uk

Accepted 11 June 2004

\section{REFERENCES}

1 Orihashi K, Matsuura Y, Sueda T, et al. Echocardiography-assisted surgery in transaortic endovascular stent grafting: role of transoesophageal echocardiography. J Thorac Cardiovasc Surg 2000;120:672-8.

2 Bruce CJ, Packer DL, O'Leary PW, et al. Feasibility study: transesophageal echocardiography with a $10 \mathrm{~F}(3.2 \mathrm{~mm})$, multifrequency $(5.5-10 \mathrm{MHz})$ ultrasound catheter in a small rabbit model. J Am Soc Echo 1999;12:596-600.

3 Kohl T, Westphal M, Strumner D, et al. Multi-modal fetal transesophageal echocardiography for fetal intervention in sheep. Circulation 2001; 104:1757-60.

4 Bruce CJ, O'Leary P, Hagler DJ, et al. Miniaturized transesophageal echocardiography in newborn infants J Am Soc Echo 2002;15:791-7.

5 Orsini AN, Kolias TJ, Strelich KR, et al. Feasibility of transoesophageal echocardiography with a ten-French monoplane probe. J Am Soc Echo 2003;16:682-7.

\section{Call for papers}

10th European Forum on Quality Improvement in Health Care 13-15 April 2005, ExCel, Docklands, London For further information on how to submit your paper please go to: http://www.quality.bmipg.com 